\title{
IONS AND ION PAIRS. THEIR MEANING AND SIGNIFICANCE IN ORGANIC REACTIONS
}

\author{
MiCHAEL SZWARC \\ Department of Chemistry, State University of New York, College of Environmental Science and Forestry, Syracuse, \\ NY 13210, USA
}

\begin{abstract}
A brief survey of the developments leading to the concept of ion pairs and of still higher ionic aggregates is followed by detailed discussion of its physical meaning. The distinction between contact ion pairs and covalently bonded molecules is clarified by comparing gaseous dissociation processes with those taking place in solution. Such a comparison reveals clearly the significance and the role of solvent molecules in homolytic and heterolytic dissociations.

In the following discussion it is pointed out that a variety of ion pairs may be formed from the pair of oppositely charged ions. An attempt is made to explain under what conditions the structurally different ion pairs may be treated as distinct species and when such a distinction fails.

Finally, examples are given to demonstrate how the structural changes of ion pairs nature may affect the rates and the equilibria of reactions in which they participate, an effort being made to provide examples pertaining to polymerization processes.
\end{abstract}

The Symposium on Ring-Opening Polymerization is concerned with reactions belonging to the class of ionic processes. The active carriers responsible for such reactions include ions, ion pairs, or their higher aggregates, and hence the discussion of the nature and behaviour of these species provides an appropriate subject for the opening remarks to this gathering. It is my intention to clarify the meaning and the significance of some concepts used in the description of ionic species, and characterize their nature and their behaviour especially under the conditions encountered in polymerizing systems.

The concept of free ions-molecules or molecular fragments endowed with some electric charge-was envisaged by Arrhenius about a century ago. He correctly deduced that neutral salts, e.g. sodium chloride, dissociate into positive cations and negative anions when dissolved in an appropriate solvent like water. Since in solution the cations and anions become independent of each other, they move in opposite directions under the influence of any electric field, the cations drifting towards cathode while anions tend to move towards anode. Their directional motion represents an electric current, and thus, the salt solution becomes conducting whereas the solvent is not. This conversion of a non-conducting solvent into a conducting solution upon dissolution of a salt is the striking verification of the idea of ionic dissociation.

Subsequent studies led to the conclusion that the ionic dissociation of inorganic salts in water is quantitative. The variation of the equivalent conductance of such solutions with salt's concentration was then accounted for in terms of electrostatic interactions between the ions, resulting in creation of "ionic atmosphere"-an increase in the local concentration of cations around an anion and vice versa.

In contrast to the behaviour of inorganic salts in water, the aqueous solutions of organic acids, bases and some other compounds revealed the phenomenon of partial ionic dissociation, its degree being governed by the masslaw as originally proposed by Arrhenius. This diverse behaviour of salts on the one hand and organic acids on the other led to the classification of the compounds capable of forming free ions into two classes, namely, strong electrolytes and weak electrolytes. The former quantitatively dissociate into ions when dissolved in an appropriate solvent, whereas the degree of ionic dissociation of the latter is given by the mass-law. This classification led Fuoss ${ }^{1}$ to suggest the term of ionophores for the compounds forming ionic crystals, and belonging therefore to the class of strong electrolytes, and the term ionogenes for those forming molecular crystals and liquids. This terminology, although highly useful, is not without a flow. There are compounds forming ionic crystals which exist nevertheless in solutions in molecular form and not as ions. For example, crystals of nitrogenpentoxide are built from positive $\mathrm{NO}_{2}^{+}$and negative $\mathrm{NO}_{3}^{-}$ions, but the solutions of nitrogenpentoxide contain the covalently bonded $\mathrm{N}_{2} \mathrm{O}_{5}$ molecules. It should be also emphasized that a hypothetical slow expansion of an ionic lattice, a process simultaneously increasing the distances between all the ions, leads ultimately to the formation of separated atoms and not ions. The formation of free ions on dissolving such a crystal in water results from hydration of the ions that prevents the redistribution of the charges occuring in the previous process.

Continuation of studies of ionic solutions led to unexpected observations. For example, Kraus ${ }^{2}$ reported that liquid ammonia solutions of typical ionophores like $\mathrm{NaCl}$ behave like those of weak electrolytes. These observations led Bjerrum ${ }^{3}$ to postulate that two oppositely charged ions may form in a solution an ion pair, a species which does not contribute to the conductance. Indeed, the early studies of ion pairs utilized the conductance data as a source of information about ion pairs. Thus, ion pairs were recognized not by their action but by the lack of action, namely, their inability to conduct electric current-surely, a highly unsatisfactory approach to characterization of a new species. This negative approach to ion pairs is reflected in a somewhat unsatisfactory definition of ion pairs originally introduced by Bjerrum and refined by Fuoss and others. ${ }^{4}$ Such a definition overemphasizes the Coulombic interaction between the positive and negative ions, and deprives ion pairs, at least to some extent, of their identity as distinct chemical species.

Positive recognition of ion pairs through their characteristic properties was reported $20 \mathrm{yr}$ after their existence 
had been postulated. The ESR studies of Weissman ${ }^{5}$ provided probably the first direct evidence for their existence. He observed the splitting of each of the 25 hyperfine lines of naphthalenide anion into four, and attributed it to the interaction of the odd electron of the radical anion with the spin $3 / 2$ of the sodium cation nucleus. This observation not only proved that the radical anion is associated with the cation but also demonstrated that such an associate has a relatively long lifetime, longer than $10^{-5} \mathrm{sec}$. This approach has been extensively used during the following years, ${ }^{6}$ and a wealth of information about the structure of ion pairs has been obtained by this technique.

Studies of ion pairs have been extensive since then and a variety of spectroscopic techniques, in addition to the ESR method, have been utilized in those investigations. Soon it became obvious that ion pairs may exist in a variety of distinct forms and thus we recognize today tight pairs, externally solvated pairs, loose pairs, solventshared pairs, etc. as well defined species, each having its own characteristic properties. Moreover, each of these species may be recognized by its specific chemical behaviour. ${ }^{7}$ There are, however, some conceptual difficulties which I now wish to clarify.

Let us start with a question: Can we consider a covalently bonded molecule and a tight, contact ion pair as two distinct species? In order to clarify this question, consider a gaseous molecule of sodium chloride. Such a molecule is characterized, at a not too high temperature, by a welldefined $\mathrm{Na}-\mathrm{Cl}$ distance corresponding to a single minimum in the potential energy curve. Moreover, it has a characteristic electron density distribution which can be deduced from appropriate experimental data or calculated by using the principles of quantum mechanics. It is desirable to remark at this place that the electron density around the sodium nucleus in $\mathrm{NaCl}$ is in fact greater than the electron density around an isolated sodium atom. Hence, it is wrong to claim that the $3 \mathrm{~s}$ electron of $\mathrm{Na}$ is transferred to $\mathrm{Cl}$ atom when the two atoms are combined into a gaseous $\mathrm{NaCl}$ molecule. Nevertheless, a gaseous $\mathrm{NaCl}$ molecule is polar, i.e. the center of gravity of the electron's cloud does not coincide with the center of gravity of the positive charges, and the direction of the resulting dipole corresponds to the direction determined by the positive NA center and the negative $\mathrm{Cl}$ center. Should we, therefore, treat the gaseous $\mathrm{NaCl}$ as a contact ion pair, $\mathrm{Na}^{+} \mathrm{Cl}^{-}$, or as a covalently bonded molecule?

The answer to this question may be obtained by considering the gaseous dissociation process resulting from a gradual stretching of the $\mathrm{Na}-\mathrm{Cl}$ bond. Such a process leads ultimately to the fission of the molecule into a neutral $\mathrm{Na}$ atom and a neutral $\mathrm{Cl}$ atom, and at no stage of this process an additional minimum in the potential curve is expected. This allows us to say that a gaseous $\mathrm{NaCl}$ is described more correctly as a covalent, although polar, $\mathrm{NaCl}$ molecule and not as a $\mathrm{Na}^{+}, \mathrm{Cl}^{-}$ion pair. However, whichever wording is used, it is obvious that we cannot imagine the existence of two distinct gaseous species, one described as $\mathrm{NaCl}$ while the other as $\mathrm{Na}^{+}, \mathrm{Cl}^{-}$.

Let us now immerse a gaseous $\mathrm{NaCl}$ in a polar liquid. The solvent molecules may organize themselves around the polar $\mathrm{NaCl}$, solvate its two ends differently and thus enhance its polarization. Such a process may lead even to some elongation of the $\mathrm{Na}-\mathrm{Cl}$ bond of the dissolved species, still keeping the partners in contact, i.e. no solvent molecules being inserted between the $\mathrm{Na}$ and $\mathrm{Cl}$. Stretching of the $\mathrm{Na}-\mathrm{Cl}$ bond in such an environment may lead to the dissociation of the dissolved molecule into a solvated $\mathrm{Na}^{+}$cation and solvated $\mathrm{Cl}^{-}$anion. As the streteching proceeds the negative charge density might increase around $\mathrm{Cl}$ and decrease around $\mathrm{Na}$ with a simultaneous tighter organization of solvent molecules around both centers. In such a case we would represent the dissolved $\mathrm{NaCl}$ molecule rather as contact ion pair than as a covalently bonded $\mathrm{NaCl}$.

It is debatable whether two distinct $\mathrm{NaCl}$ species may exist in a solution, one tight, less polar and "no-solvated", the other looser, but still a contact one and well "solvated." Conceptually such a situation may be envisaged. It should be stressed, however, that the feasibility of such a phenomenon hinges on the presence of solvent molecules surrounding the dissolved species and providing two different local environments. Such a situation is impossible in the gaseous phase.

The problem of co-existence of a covalently bonded molecule with a contact ion pair becomes even more interesting when we deal with carbanions or carbonium ions. Let us consider, for example, triphenylmethyl chloride. The covalent molecule has an $\mathrm{sp}_{3}$ hybridisation and tetrahedral configuration around the central carbon atom, whereas a hypothetical contact ion pair should have an $\mathrm{Sp}_{2}$ hybridization and coplanar $\mathrm{C}-\mathrm{Ph}$ bonds with the $\mathrm{Cl}^{-}$ion located above the plane defined by the three $\mathrm{C}-\mathrm{Ph}$ bonds. Thus, the difference in the geometry permits us to visualize a covalent $\mathrm{CPh}_{3} \mathrm{Cl}$ and a contact $\mathrm{CPh}_{3}{ }^{+}, \mathrm{Cl}^{-}$ion pair as two distinct species which could coexist even in the gaseous phase.

We may consider now the distinction between contact ion pair and an externally solvated contact ion pair. Such a distinction is meaningful when the solvation involves a solvating agent different from the bulk solvent. For example, we determined ${ }^{8}$ the equilibrium established in diethyl ether between the tight sodium naphthalenide ion pair, $\mathrm{N}^{\top}, \mathrm{Na}^{+}$, and the externally THF solvated pair formed in this system when relatively small amounts of THF were added to the diethyl ether. Interestingly, the investigated equilibrium results from the reaction

$$
\mathrm{N}^{\top}, \mathrm{Na}^{+}+2 \mathrm{THF} \rightleftarrows \mathrm{N}^{\top}, \mathrm{Na}^{+}(\mathrm{THF})_{2} .
$$

However, such a distinction between two species becomes meaningless when the concentration of the solvating agent is too high. The last statement raises the general question: what is meant by a distinct species in a solution?

A simple treatment of elementary gaseous reactions avoids the distinction between those subspecies that differ from each other by the magnitude of their linear momentum or by the state of their rotation and vibration, although more refined treatments are concerned with the distinction between various rotational and vibrational subspecies. The various subspecies contribute to a different degree to the observed properties of the investigated molecules, and these differences may be taken into account by attributing to those molecules average properties arising from appropriate averaging procedures.

In a liquid the properties of the dissolved molecules are modified by their dynamic interaction with the surrounding solvent molecules. Since the system is undergoing a Brownian motion, these interactions fluctuate and therefore the momentary state of each invividual molecule is modulated. The averaging procedure takes cognizance of these modulations and a thermodynamic treatment accounts for the resulting average changes in the properties of dissolved molecules by introducing the concepts of 
activities and activity coefficients. Such an approach is fully justified when the interactions between the solute and solvent are weak. Although this approach is still formally correct even for strongly interacting systems, it might seem intellectually unsatisfactory. Indeed, the concept of activities hides all the interesting details of solute-solvent interactions which are responsible for a variety of structures that deserve thorough examination. Such structures often become well defined and long-lived in solution of ions and ion pairs in polar liquids because the intervening forces are strong.

Let us consider the molecular patterns involving the ion in question, or an ionic aggregate, in conjunction with the neighbouring molecules of the solvent and some added solvating agent, provided that the latter are present in the studied solution. Due to the Brownian motion, the vigor of which depends on temperature, no pattern lasts long. Usually it persists for less than $10^{-10} \mathrm{sec}$, although some of its basic features may be retained for a relatively long time. A set of patterns sharing such a common basic feature describes what shall be denoted as a species in liquid phase. The patterns composing such a set are easily interchanged, one being readily transformed into another. Nevertheless, the identity of such a defined species is preserved as long as the common basic features of the patterns are retained, but whenever the basic features are modified or destroyed, the respective species loses its original identity.

A solution is composed only of one type of ionic species if all the molecular patterns formed around the dissolved ionic aggregate belong to a single set, in other words, if all possess the same basic features. What constitutes such a species depends, however, on our definition of the basic features. A more rigorous and detailed specification may modify our statement and force us to treat the same solution, supposedly composed of one solute, as a mixture of two or more different kinds of ionic species.

This ambiguity makes one wonder whether the proposed description of ionic species is justified and useful. To clarify this point, let us consider a multicomponent solution, a system involving molecular patterns assigned to several sets each characterized by its own basic features. Such an assignment is useful provided the respective sets are not overlapping, because only then each molecular pattern is unambiguously attributed to one, and only one, set. The nonoverlap condition introduces an important restriction upon our choice of the basic features that are appropriate for defining a set. If these restrictions are met, the resulting nonoverlapping sets define a mixture of distinct species amenable to the conventional thermodynamic treatment.

Let us now consider a pattern developed in a solution around an ion or an ionic aggregate. Although it varies with time, its basic feature may be retained for a relatively long period thus permitting its identification with a kind of species. A sufficiently powerful fluctuation may suddenly transform it into a new pattern associated with another set. Such an event is interpreted as a reaction: species $i \rightarrow$ species $j$. In this context such concepts as the mole fraction, $\gamma_{i}$, of species $i$, their lifetime, $\tau_{i}$, and their rate constant, $k_{i j}$, of conversion into species $j$, become selfexplanatory.

The sharp differentiation between the distinct species, say $i$ and $j$, is the consequence of the nonoverlapping nature of the sets. However, the requirement of continuity implies that a transition from a pattern belonging to set $i$ into a pattern associated with set $j$, proceeds through some "freak" patterns not included in any of the recognized sets. The "freak" patterns, although excluded from our classification, appear in the solution under investigation. If their occurrence is rare, their contribution to the properties of the system is negligible and their omission is justified. Under such conditions the distinction between species $i$ and $j$ is not only permissible but also profitable.

This additional restriction of our definition of distinct species deserves further examination. The "freaks" represent the highly improbable patterns, the low probability of their occurrence caused often, although not always, by the high energy of their formation. As the thermodynamic conditions vary, for example, as the temperature rises, the proportion of the "freaks" may increase and reach a level when their omission is no longer justified. Our differentiation between the previously distinct species breaks down, and then they have to be treated as one kind only. The following two examples may clarify these ideas.

Two types of ion pairs may be present in a solution. Ions of one pair are virtually in contact with each other, and such a pair would be denoted as $t$. Alternatively, the two ions could be separated by one or two solvent molecules and kept apart at an average distance $r$. These looser pairs are referred to as I pairs. Since either pair may vibrate and the amplitude of the vibration increases with rising temperature, the distinction between the two kinds of pairs could be justified at low temperatures but it might be blurred at higher temperatures when the vibrations are strong and cause an overlap of the respective patterns. Thus the differentiation may fail even if the extrapolation from the low temperature data indicates that the concentrations of both kinds of pairs should be finite at high temperature. Hence the failure of differentiation between the two kinds of ion pairs is not caused by the disappearance of one type of pair from the solution. It arises from the breakdown of the concept of two types of pairs, a concept deprived of its justification in this temperature range.

Differentiation of free ions from ion pairs is unambiguous in a dilute salt solution. While the former are randomly distributed throughout the liquid and kept, on the whole, far away from one another, the latter represent two ions kept closely together. The ratio of free ions to ion pairs may be kept constant as the solution is concentrated, provided that the temperature is varied judiciously. However, at a sufficiently high concentration of salt the average distance between the free ions becomes too small to justify the distinction between free ions and ion pairs and then it is no longer possible to maintain the concept of free ions and ion pairs as two distinct species. Surely, it is senseless to differentiate between free ions and ion pairs in a molten salt.

The two examples and the preceding discussion should clarify the meaning, as well as the limitations, of the concepts of ionic species. Discussion of ionic reactions becomes clearer when the reagents are described in these terms. The treatment of the whole field becomes harmonious when the physical observations are combined with the kinetic data discussed here. Moreover, much is gained by discussing the available observations in terms of the virtually invariant properties of different ionic species and the variable composition of the solution.

The simultaneous coexistence of several ionic species introduces specific complications in studies of mechanism of chemical reactions. For example, the apparent propagation constant of anionic polymerization of styrene in 
tetrahydrofuran increases with dilution of living polymers, ${ }^{9}$ and this phenomenon arises from the participation of free living polystyryl anions, as well as their ion pairs, in the polymerization process. The former propagate with a rate constant of about $65,000 \mathrm{M}^{-1} \mathrm{sec}^{-1}$, whereas the latter grow with a rate constant which varies from $25 \mathrm{M}^{-1} \mathrm{sec}^{-1}$ when the pair involves $\mathrm{Cs}^{+}$cations to about $180 \mathrm{M}^{-1} \mathrm{sec}^{-1}$ for the lithium ion pairs. This example illustrates the great difference in the reactivity of free ions and of ion pairs and shows also how the nature of cation affects the reactivity of ion pairs.

It is important to stress at this junction that it is not necessary to expect always a higher reactivity of free ions and a lower for ion pairs. Although this gradation is shown in a number of systems, some being discussed in this symposium, others like the polymerization of epoxides or propylene sulfide to be discussed here show an opposite trend-free ions being less reactive than ion pairs.

The nature of solvent is again important in determining the rate of propagation. For example, sodium polystyrene ion pairs propagate with a rate constant of about $3 \mathrm{M}^{-1} \mathrm{sec}^{-1}$ in dioxane but the rate constant is $3600 \mathrm{M}^{-1} \mathrm{sec}^{-1}$ in dimethoxyethane. Such a great change in the reactivity is attributed to the change in the structure of ion pairs. Tight pairs are present in dioxane solution but the greatly more reactive loose pairs dominate in dimethoxyethane. The change in the structure of ion pairs is responsible for the dramatic effects observed on the propagation of sodium polystyryl in tetrahydropyrane ${ }^{10}$ upon the addition of small amounts of tetraglyme. In the absence of the glyme the propagation proceeds with a rate constant of about $12 \mathrm{M}^{-1} \mathrm{sec}^{-1}$, but it increases a thousandfold in the presence of the glyme.

It should be stressed again that the tight pairs are in some reactions more reactive than the loose ion pairs and there are good reasons why the reverse order of reactivities is observed.

When an ion pair is solvated by an agent present at low concentration in the reacting system, two distinct situations may be observed. The agent may solvate externally the ion pair, or it may separate the two associated ions and form an agent-separated-loose pair. Under proper conditions both types may be present simultaneously in a solution and then one may study the equilibrium

$$
\mathrm{A}^{-}, \mathrm{C}^{+}, \mathrm{X} \rightleftarrows \mathrm{A}^{-}, \mathrm{X}, \mathrm{C}^{+} .
$$

A first example of such a system was reported by Slates and Szwarc, ${ }^{11}$ and other similar systems were discovered later.

While the rate of propagation of sodium living polystyrene in tetrahydrofuran increases on dilution, the reverse is observed in the polymerization of its barium salt. This result is due to the participation of "triple ions" involving barium cation and three polystyryl anions, and the kinetic, as well as the conductance data are quantitatively accounted for by the proposed mechanism. ${ }^{12}$
The nature of ion pairs may exert a profound effect on the stereochemistry of ionic polymerization. The effect of $\mathrm{Li}$, as distinct from other alkali metals, responsible for the 1,4-cis structure of polydiene is a striking example of directed stereochemistry arising from the nature of cation in the ion pair. Examples of the effect of solvent upon the stereochemistry of acrylate polymerization are provided, e.g. by the studies of Schuerch, and the same group demonstrated the effect of counter anion on the glycosidic linkage of polysaccharides.

Finally, I cannot resist mentioning one more example, not pertaining to polymerization processes, which shows how enormously the equilibrium of a system involving ionic species is affected by their structure. The equilibrium constant of disproportionation of tetracene radical anions into dianions, i.e.

\section{Tetracene ${ }^{-}$, Cat $^{+} \rightleftarrows$ Tetracene + Tetracene $^{2-}, 2$ Cat $^{+}$,}

varies by 11 powers of ten, being $\sim 10^{-10}$ for the lithiumtetrahydrofuran system and $\sim 10^{1}$ in lithium-diethylether system. $^{13}$

I believe that these examples clearly demonstrate that studies of ionic reactions in general, and ionic polymerization in particular, cannot be comprehended and quantitatively accounted for without understanding the structure, properties, and interrelations (equilibria and rates of conversion) of the various ionic species that participate in the investigated precesses.

\section{REFERENCES}

1. R. M. Fuoss, J. Chem. Educ. 32, 527 (1955).

2. R. M. Fuoss and C. C. Kraus, J. Am. Chem. Soc. 55, 476 (1933).

3. N. Bjerrum, KgH. Danske Viden. Selsk. 7, 9 (1926).

4. M. Szwarc, Carbanions, Living Polymers, and Electron Transfer Processes. pp. 218-225, Interscience, New York (1968), for the discussion of this subject.

5. (a) F. C. Adams and S. I. Weissman, J. Am. Chem. Soc. 80, 1518 (1958). (b) N. M. Atherton and S. I. Weissman, Ibid, 83, 1330 (1961).

6. Chapters V, VII and VIII in; Ions and Ion Pairs in Organic Chemistry. (Edited by M. Szwarc), Vol. 1, John Wiley, New York (1972).

7. Ions and Ion Pairs in Organic Chemistry. (Edited by M. Szwarc), Vol. 2. John Wiley, New York (1974).

8. Lydia Lee, R. Adams, J. Jagur-Grodzinski and M. Szwarc, J. Am. Chem. Soc. 93, 4149 (1971).

9. D. N. Bhattacharyya, C. L. Lee, J. Smid and M. Szwarc, J. Phys. Chem. 69, 612 (1965)

10. M. Shinohara, J. Smid and M. Szwarc, J. Am. Chem. Soc. 90, 2175 (1968); Chem. Comm., Lond. 1232 (1969).

11. R. V. Slates and M. Szwarc, J. Am. Chem. Soc. 89, 6043 (1967).

12. B. De Groof, M. Van Beylen and M. Szwarc, Macromolecules 8, 396 (1975).

13. G. Levin and M. Szwarc, J. Am. Chem. Soc. (1976) In press. 